\title{
Münchener Kommentar zum BGB
}

\author{
Inhaltsübersicht
}
A. Äußere Entwicklung des Kommentars: Von der ersten Auflage zur fünften Auflage
B. Reformbedingte Wandlungen
C. Innere Entwicklung des Kommentars
D. Europäisierung des Privatrechts

\section{A. Äußere Entwicklung des Kommentars: Von der ersten zur fünften Auflage}

Daß ohne verlegerischen Elan und wirtschaftliche Risikobereitschaft Großkommentare nicht möglich sind, ist angesichts der hohen finanziellen Investitionen, die mit einem solchem Projekt verbunden sind, jedermann einsichtig. Ich war mir daher im November 1970 nach Annahme des Rufs an die Freie Universität Berlin des Wagnisses bewußt, das ich dem Inhaber eines Verlages aufdrängte, als ich die Idee an ihn herantrug, einen neuen zehnbändigen Kommentar zum BGB herauszugeben. Mein ursprünglicher Gedanke war es, den neuen Großkommentar „,Berliner Kommentar zum BGB“" zu nennen. Ich schrieb daher den Inhaber des damals noch selbständigen Vahlen-Verlages in Berlin, Herrn Gundlach, den ich seit meiner Mitarbeit am Lehrbuch des Arbeitsrechts von Hueck/Nipperdey gut kannte, an und erinnerte ihn an die große Tradition seines Verlages bei der Herausgabe von Kommentaren, u.a. zum HGB, zum Aktienrecht und zum BGB. ${ }^{1}$ Eher vollmundig-verwegen als empirisch abgesichert bekundete ich, daß es aus der Sicht von Wissenschaft und Praxis nunmehr an der Zeit sei, einen neuen wissenschaftlichen Kommentar herauszugeben, der nicht wie alle anderen Erläuterungswerke zum BGB noch die Schlacken seiner Entstehungszeit, nämlich das erste Jahrzehnt nach Inkrafttreten des BGB, mitschleppe, sondern der, frei von abgestorbenen historischen Problemlagen, Beispielen und Meinungsständen, neu beginne und das Privatrecht nach Inhalt und Methode zeitgerecht kommentiere.

Herr Gundlach nahm diese Idee mit großer persönlicher Begeisterung auf. Bevor es aber zu definitiven vertraglichen Absprachen kam, verkaufte er den Vahlen-Verlag - selbstverständlich mit dem Konzept eines neuen Großkommentars in der Schublade - an den Beck-Verlag. Ich habe dann Ende Februar 1972 Herrn Dr. Beck angesprochen und für die Edition eines neuen Großkommentars zum BGB geworben. Herr Dr. Beck griff die Idee mit Schreiben vom 28. März 1972 -

${ }^{1}$ Vgl. nur Schlegelberger/Vogels, Erläuterungen zum BGB, 1939; Schlegelberger, HGB, 1. Aufl. 1939, zuletzt: HGB, 5. Aufl. 1973 ff.; Schlegelberger, AktG, 1. Aufl. 1937, zuletzt: Geßler/Hefermehl/Eckardt/Kropf, Aktiengesetz, 1973 ff. 
mit der ihm eigenen Vorsicht und Umsicht - auf. Er hatte, wie er mir später sagte, das Projekt durchrechnen lassen und reduzierte aus Kostengründen das Volumen von zehn Bänden auf sechs Bände und wünschte sich einen zweiten Herausgeber aus der Praxis, um der Gefahr vorzubeugen, daß der Kommentar zu theorielastig werde. Herr Dr. Beck schrieb mir damals:

„Die Erfolgsaussichten des Kommentars dürften entscheidend davon abhängen, ob er sich besonders für die richterliche und anwaltschaftliche Praxis eignet. Der Absatz, der bei den wissenschaftlichen Instituten zu erreichen ist, fällt demgegenüber nicht wesentlich ins Gewicht."

Herr Dr. Beck leitete daraus als zwingende Folge ab, daß die Autoren ,etwa zu gleichen Teilen“ aus der Universität und aus der Praxis kommen sollten. Das Ehescheidungsrecht war damals gerade durch eine Kommission unter der Leitung des baden-württembergischen Ministerialdirektors Dr. Kurt Rebmann, der damals noch nicht das schwere Amt des Generalbundesanwalts angetreten hatte, unter Abkehr vom Verschuldensprinzip grundlegend reformiert worden. Da ich davon wenig verstand, war ich froh, daß Herr Dr. Beck in Herrn Dr. Rebmann einen äußerst tatkräftigen, hochmotivierten und bei Ahndung von Terminüberschreitungen auch gefürchteten Mitherausgeber fand, der zugleich die Bandredaktion für das Familienrecht übernahm. 1978 war dann der Startbeginn der ersten Auflage mit dem neuen Familienrecht. Inzwischen ist als weiterer Herausgeber aus der Praxis Herr Professor Dr. Rixecker, der Präsident des OLG Saarbrücken, hinzugetreten.

Neben den Herausgebern übernahmen die Bandredakteure für die Qualität der in dem von ihnen betreuten Band abgedruckten Manuskripte eine wichtige Mitverantwortung. Inzwischen ist die erste Generation der Bandredakteure, mit Ausnahme der Herren Kollegen Westermann und Sonnenberger, mit dem Erscheinen der vierten Auflage, einige auch bereits mit der dritten Auflage, ausgeschieden. Ihnen gilt an dieser Stelle der große Dank des Verlages und der Herausgeber. Ohne ihre Übersicht, ihr Problembewußtsein und ihre Mühen bei der Durchsicht der Manuskripte und bei vielen Gesprächen mit einzelnen Autoren wäre der Münchener Kommentar zum BGB keine so große Erfolgsgeschichte für den Verlag geworden. Gedankt sei an dieser Stelle daher insbesondere den Herren OLG-Präsident a.D. Professor Dr.h.c. Heinrichs (Bandredakteur von Bd. II der 1.-3. Auflage; Nachfolger: Bundesrichter Professor Dr. Krüger), Professor Dr. Dres. h.c. Ulmer (Bandredakteur von Bd. V der 1.-4. Auflage; Nachfolger: Professor Dr. Habersack), Bundesrichter a.D. Professor Quack (Bandredakteur von Bd. VI der 1.-3. Auflage), VorsR BGH Dr. Rinne (Bandredakteur von Bd. VI der 4. Auflage) und Bundesrichter a.D. Dr. Manfred Skibbe (Bandredakteur von Bd. IX der 1.-3. Auflage). Bei der fünften Auflage, die mit Bd. I/1 (Allgemeiner Teil) und Band I/2 (Antidiskriminierungsrecht) soeben auf den Markt gekommen ist, werden folgende Bandredakteure neben den Herausgebern Freude und Last der Verantwortung tragen:
Bd. I ( $\$ 1-240$ und AGG):
Professor Dr. Dr. Dr.h.c. Franz Jürgen Säcker
Bd. II (\$ 241-432):
Professor Dr. Wolfgang Krüger, Richter am Bundesgerichtshof
Bd. III (\$§ 433-606):
Professor Dr. Harm Peter Westermann 
Bd. IV (\$§ 607-704):

Bd. V (\$§ 705-853):

Bd. VI (§§ 854-1296):

Bd. VII (§§ 1297-1588):

Bd. VIII (§§ 1589-1921):

Bd. IX (§§ 1922-2385):

Bd. X (Art. 1-46 EGBGB):

Bd. XI (Art. 50-245 EGBGB):
Professor Dr. Martin Henssler

Professor Dr. Martin Habersack

Dr. Reinhard Gaier, Richter am Bundesverfassungsgericht

Beatrix Weber-Monecke, Richterin am Bundesgerichtshof

Professor Dr. Dieter Schwab

Dr. Gerhard Schlichting, Richter am Bundesgerichtshof

Professor Dr. Dr. h.c. Hans Jürgen Sonnenberger

Professor Dr. Dr. h.c. Hans Jürgen Sonnenberger

In alljährlichen Treffen werden im Kreis der den Kommentar betreuenden Lektoren des Beck-Verlages (zunächst Herr Dr. Letzgus, dann Herr Dr. Huber, heute Herr Dr. Weber und - von Anfang an dabei - Frau von Bonhorst sowie später auch Frau Koppold), der Bandredakteure und der Herausgeber alle wichtigen Fragen und Probleme besprochen und entschieden. Natürlich sind Herausgeber und Bandredakteure, auf sich allein gestellt, nichts wert, wenn nicht eine Fülle fachlich hoch versierter, von Arbeitsfreude gepackter und Freizeiteinbußen akzeptierender Autoren die Hauptlast der Arbeit getragen und die Erläuterungen von Auflage zu Auflage aktualisiert, verbessert und verfeinert hätte. Die große Zahl der Autoren verbietet es, an dieser Stelle jedem einzelnen von ihnen unter Nennung seines Namens und seiner speziellen Verdienste zu danken, zumal der Dank nicht nur an die aktiven, sondern auch an die inzwischen in den Ruhestand getretenen Autoren zu richten wäre. Unser Dank und unser Gedenken gilt dabei in besonderer Weise denen, die nicht mehr unter den Lebenden weilen, aber ohne deren Mitwirken der Kommentar in seiner Qualität nicht denkbar gewesen wäre.

$\mathrm{Zu}$ einem Zeitpunkt, da niemand mehr ernstlich an eine Wiedervereinigung glaubte, entschied Herr Dr. Beck, den Kommentar nicht im Vahlen-Verlag, sondern als neues Flagschiff des Beck-Verlages unter der Bezeichnung „Münchener Kommentar zum BGB“" erscheinen zu lassen. Der Erfolg des Kommentars führte in der Folge dann zu einer ganzen Familie erfolgreicher Münchener Kommentare. ${ }^{2}$ Ich selber konnte mit der Namensänderung gut leben, da ich 1973 von der Freien Universität Berlin an die Universität Hamburg gewechselt war. Nicht ganz glücklich war ich allerdings über die meinem Konzept widersprechende Beschränkung auf sechs Bände. Diese unterliefen wir, vom damaligen Lektor Dr. Letzgus klammheimlich unterstützt, dadurch, daß wir in der ersten Auflage zwar nur bis sechs zählten, dafür aber das für die Praxis besonders wichtige Besondere Schuldrecht auf die Bände III/1 und III/2 aufteilten. In der zweiten Auflage erweiterten wir dann - aufgrund des Erfolges der ersten Auflage nunmehr mit

2 Vgl. die Münchener Kommentare zum HGB, zur ZPO, zum Aktienrecht, zum StGB, demnächst zum Unlauterkeitsrecht, zum Deutschen und europäischen Wettbewerbsrecht, zum Versicherungsvertragsrecht usw. 
Zustimmung des Verlegers - das Besondere Schuldrecht auf drei Bände, das Familienrecht und das EGBGB ebenfalls jeweils auf zwei Bände und hatten damit sogar die Zahl von elf Bänden erreicht. Eine in der Sache richtige Konzeption läßt sich nicht unterdrücken. Die Natur der Sache bricht sich Bahn und sprengt mitunter alle kaufmännischen Vorgaben des Verlegers, auch wenn sein Wille für die Autoren in aller Regel unbeugsamer ist als der Wille des Gesetzgebers. ${ }^{3}$ Wir konnten den vorgegebenen Umfang des Gesamtwerks daher nur sprengen, weil die Käufer uns gefolgt sind, alle Bände ordentlich gekauft und damit den Herausgebern des Münchener Kommentars eine schimpfliche Entlassung durch den Verleger erspart haben.

Mit der vierten Auflage hat der Kommentar aus der Sicht der Herausgeber und des Verlages seine optimale und sachgemäße Größe erreicht. Die Quantität ist im Interesse einer Qualitätsverbesserung nicht mehr auszubauen; es gilt vielmehr, das erreichte Qualitätsniveau mit knapperer Übersicht über den aktuellen Meinungsstand in Wissenschaft und Praxis und mit präziser Stellungnahme zu sichern sowie durch Gewinnung neuer Mitarbeiter, die neue Gedanken einbringen, kontinuierlich weiter zu verbessern und den Umfang der Bände durch Richtlinien, Sollvorgaben und individuelle Überzeugungsgespräche mit einzelnen Autoren, die die Sollvorgaben für ihre Abschnitte überschreiten, in den festgelegten Grenzen zu halten.

Von Anfang an war es das Ziel, für die Erläuterung der einzelnen Abschnitte junge Wissenschaftlerinnen und Wissenschaftler zu gewinnen, die sich als Spezialisten auf dem betreffenden Gebiet, z. B. durch eine Habilitationsschrift, hervorgetan hatten und von ihrem Lebensalter her in der Lage waren, an mehreren Auflagen mitzuwirken. Dies sollte sicherstellen, daß der Kommentar nicht als Synthese aus der vergleichenden Zusammenschau vorhandener alter Erläuterungswerke entstand, sondern in kritischer Auseinandersetzung mit Rechtsprechung und Literatur neu konzipiert und geschrieben wurde. Er sollte die Erkenntnisse wissenschaftlicher Forschung fall- und praxisnah präsentieren und offene Probleme unter Berücksichtigung der Entstehungsgeschichte und der legislativen Ratio der Vorschrift lösen. Der Jurist hat die Pflicht - so Thibaut in seiner ,,Theorie der logischen Auslegung des römischen Rechts“ (1806) -, „nicht bloß dem Buchstaben (dem Wortverstand) des Rechts zu folgen, sondern zugleich mit dem Geist desselben Rücksicht zu nehmen." Mich selber leitete dabei - als Schüler Nipperdeys das Wort, das dessen Lehrer Hedemann der Habilitationsschrift Nipperdeys über „Kontrahierungszwang und diktierter Vertrag“ (1920) vorangestellt hatte:

„Es kann keinem Zweifel unterliegen, daß das rechtswissenschaftliche Schrifttum in Unordnung geraten ist. Eine neuartige, oft flüchtige und flache, auf Handlichkeit zugeschnittene Schriftenart hat den vor dreißig oder fünfzig Jahren durchaus vorherrschenden Typus der gediegenen, wohldurchdachten Einzelschrift aus dem Felde geschlagen. Der Kommentar triumphiert, aber schon sinkt er hinunter zur bloßen rasch verfaßten, auf schnellsten äußeren Erfolg abgestimmten Handausgabe. Das kann nicht gut sein.“

${ }^{3}$ Vgl. dazu näher Säcker, Einleitung Münchener Kommentar zum BGB, 5. Aufl. Bd. I 2006, Rn. $115 \mathrm{ff}$. sowie ders., L'harmonisation du droit communautaire privé avec les droits privés nationaux et les méthodes classiques de l'interprétation juridique, 2004, S. 33 ff. u. $60 \mathrm{ff}$. 
Wir wollten keinen flachen Handkommentar, sondern einen gediegenen, wohldurchdachten Großkommentar ins Leben rufen, der die Praxis befruchtet und vom Richter konsultiert wird, bevor er entscheidet. Wir haben uns aus diesem Grunde bemüht, neben praxisorientierten Theoretikern aus dem Bereich der Wissenschaft theorieinteressierte Praktiker zu gewinnen, die durch ihre konkrete berufliche Tätigkeit mit dem Rechtsgebiet eng vertraut waren, das sie erläutern sollten. Der Kommentar sollte kein dogmatisch geprägtes Handbuch und kein Lehrbuch mit monographischem Charakter werden, sondern ein vom Telos der Vorschrift geleitetes, in Kenntnis des konkreten Wirklichkeitsausschnitts geschriebenes Werk. Die angesprochenen Autoren haben sämtlich engagiert mitgemacht und dem Kommentar die Treue gehalten. Allerdings stellte sich in der vierten und in Vorbereitung der fünften Auflage das Problem, in großem Umfang neue Autoren an Stelle altersbedingt ausgeschiedener Mitarbeiter zu gewinnen, die sich mit den Zielen des Münchener Kommentars in gleicher Weise identifizieren wie die ausgeschiedenen Autoren. Dies ist bis heute trotz der großen Zahl der Mitarbeiter hervorragend gelungen. An der vierten Auflage haben immerhin 124 Experten aus Wissenschaft und Praxis mitgearbeitet, bei der fünften Auflage werden es nicht weniger sein.

\section{B. Reformbedingte Wandlungen}

Der Kommentar hat in den vergangenen 30 Jahren seines Bestehens vor allem durch die Gesetzgebung grundlegende Wandlungen erfahren. Der erste gravierende Einschnitt für den Münchener Kommentar war die Herstellung der Rechtseinheit zwischen den beiden Teilen Deutschlands, die vor völlig neue Aufgaben stellte. Der Münchener Kommentar hat das Privatrecht des Einigungsvertrages unmittelbar nach dem Beitritt der DDR zur Bundesrepublik Deutschland in einem erfolgreich verkauften Sonderband umfassend erläutert und die der Herstellung der Rechtseinheit, aber auch dem Rechtsfrieden in den neuen Bundesländern dienenden Gesetze (u.a. Sachen- und Schuldrechtsbereinigungsgesetz) eingehend dargestellt. Der nächste gravierende Einschnitt vollzog sich mit der vierten Auflage, die in wichtigen Partien von der zunehmenden Europäisierung, aber auch Internationalisierung des Privatrechts, insbesondere des Vertrags- und Wirtschaftsrechts geprägt ist. Der elfte Band der vierten Auflage ist deshalb zu einem umfassenden Erläuterungswerk des internationalen Handels-, Gesellschafts-, Wirtschafts- und Insolvenzrechts ausgebaut worden, das in dieser umfassenden Konzentration ohne Vorbild ist und die übrigen Münchener Kommentare entlastet.

Das Schuldrecht bedurfte in der vierten Auflage grundlegender Umgestaltung und wird in der fünften Auflage durch Einarbeitung der neu vorliegenden Rechtsprechung nochmals wachsen. Immer mehr Normen des Schuldrechts, insbesondere des Verbraucherschutzrechts, haben europäischen Ursprung und unterstehen dem Gebot der richtlinienkonformen Auslegung. Dieses soll sichern, daß die Normen EG-rechtlichen Ursprungs in allen Mitgliedstaaten der EG einheitlich 
angewandt werden. Waren die ersten drei Auflagen durch eine behutsame Entwicklung des Norminhalts mit Hilfe einer ,vertikalen“ dogmengeschichtlichen Durchdringung des Stoffs gekennzeichnet, so ist die vierte Auflage durch eine „horizontale“ Erweiterung des Blickwinkels durch eine rechtsvergleichende Heranziehung europäischen Privatrechts geprägt.

Besonders deutlich wurde der Konflikt zwischen dem Beharren auf der historisch gewachsenen nationalen Lösung und dem teleologischen Expansionsdrang des europäischen Rechts bei der aktuellen Diskussion um die Umsetzung der EG-Gebrauchsgüterkauf-Richtlinie. Während nach Auffassung des Bundesjustizministeriums als Folge der Umsetzung nicht neue isolierte „Inseln europäischen Rechts“" entstehen sollten, sondern ein „Festlandsockel“ einheitlichen Schuldrechts, wollten viele Wissenschaftler, zunächst am alten Schuldrecht festhalten und die Zeit nutzen, um eine umfassende, europäisch geprägte Schuldrechtskodifikation vorzubereiten. ${ }^{5}$

Nun lassen sich die heftigen Diskussionen um eine Schuldrechtsreform nicht auflösen in einen Konflikt zwischen Traditionalisten, die sich auf Savigny berufen und die Zeit für eine große Schuldrechtsreform noch nicht für reif halten, und den Anhängern einer an Thibaut anknüpfenden, ${ }^{6}$ nach Rechtseinheit rufenden Schuldrechtsreform, die ein europäisches Privatrecht aus einem Guß als übergreifendes System wollen, das ,altes“ deutsches Schuldrecht ablöst. Daß die Mehrzahl der Privatrechtslehrer, ,genervt“ durch interventionistisch geschaffenes, häufig wenig ausdiskutiertes punktuelles europäisches Privatrecht ${ }^{7}$, einen grundlegenden Eingriff in das aus dem BGB entstandene, wissenschaftlich durchstrukturierte und vertraute Schuldrecht ablehnte, ist nicht nur emotional, sondern auch von der Sache her verständlich, wenn man an die lange Beratungsphase des BGB denkt und sie mit der kurzen Zeit vergleicht, die zur konkreten Diskussion des Referentenentwurfs zur Schuldrechtsreform zur Verfügung stand.

Ein völlig neues Problem stellt sich in der fünften Auflage mit der Integration des Gleichbehandlungsgesetzes ${ }^{8}$ und der am 21. Dezember 2004 in Kraft getretenen, bis zum 21. Dezember 2007 von den Mitgliedstaaten umzusetzenden Richtlinie zur Verwirklichung des Grundsatzes der Gleichbehandlung von Männern und Frauen beim Zugang zu und bei der Versorgung mit Gütern und Dienstleistungen $^{9}$ in das Wertungsgefüge des Privatrechts. Das Antidiskriminierungsrecht ist zwar entgegen ursprünglichen Überlegungen in der Amtszeit der früheren Bundesjustizministerin Prof. Dr. Däubler-Gmelin nicht in das BGB eingearbeitet, ${ }^{10}$ son851.

${ }^{4}$ Vgl. Rittner, Das Gemeinschaftsprivatrecht und die europäische Integration, JZ 1995, 849,

${ }_{5}^{5}$ Vgl. dazu den ein Jahr nach Inkrafttreten der Schuldrechtsreform erschienenen Bd. 2a der vierten Auflage des Münchener Kommentars zum BGB.

${ }^{6}$ Vgl. dazu Hattenhauer, Thibaut und Savigny. Ihre programmatischen Schriften, 1973; Coing, Europäisches Privatrecht 1800-1914, Bd. II, 1989, S. 15 ff.

${ }^{7}$ Vgl. dazu die Einleitung zur 4. Aufl. des Münchener Kommentars zum BGB, Bd. I, Rn. $188 \mathrm{ff}$.

8 Vgl. BGBl. I 2006 S. 1897 ff.; dazu Thüsing, Einl. Bd. I/2.

${ }^{9}$ ABl. EG Nr. L 373, S. 37 v. 21. 12. 2004.

${ }^{10}$ Auch das AGB-Recht ist erst mit der im Zeichen der Verbesserung des Verbraucherschutzes stehenden Schuldrechtsreform in das BGB eingearbeitet worden. Vgl. zur Entstehungsge- 
dern in einem eigenen Gesetz außerhalb des BGB geregelt worden. Es ist aber, da es die Abschlußfreiheit im Geschäftsverkehr weitreichend einschränkt, ein die normativen Ordnungsstrukturen des Vertragsrechts grundlegend veränderndes Recht. Die vor Motivzensur schützende normative Leitidee der Privatautonomie: „Pro ratione stat voluntas!“ („Es gilt der freie Wille ohne Zensur seiner Vernünftigkeit.") verliert im Schuldrecht ihre Geltung; sie wird von einer umfassenden „Anständigkeitskontrolle“ abgelöst. ${ }^{11}$ Die Vertragsfreiheit unterliegt damit - weit über $\S 138$ BGB hinausgehend - staatlich definierten Tugendvorgaben, die als Richtschnur privatautonomen Handelns dienen sollen. Abweichendes Verhalten bedarf der sachlichen Rechtfertigung durch das sich auf Vertragsfreiheit berufende Privatrechtssubjekt, um wirtschaftlichen Nachteilen zu entgehen. Weltanschaulich geprägte Meinungsäußerungen, die durch Art. 5 Abs. 1 GG geschützt sind, werden negativ sanktioniert durch einen über die neuen Antidiskriminierungsverbote vermittelten Kontrahierungszwang bzw. Schadensersatz. Die fünfte Auflage steht damit vor der schwierigen Aufgabe, das gesetzlich veränderte Verhältnis von Freiheit und Gleichheit fallgruppenbezogen neu auszutarieren.

\section{Innere Entwicklung des Kommentars}

Der Münchener Kommentar konnte eine über die äußere Einheitlichkeit und Harmonie des Druckbildes hinausgehende ,,innere Einheit“" nur erreichen, indem die Herausgeber zusammen mit den Bandredakteuren im Sinne guter ,,corporate governance" von Anfang an über redaktionelle Richtlinien für die Methodik der Darstellung einen verbindlichen Ordnungsrahmen vorgaben, und zwar sowohl bezogen auf die Interpretationsprinzipien als auch auf die sprachliche Gestaltung.

Nach den redaktionellen Leitlinien hat der Autor den Inhalt der zu erläuternden gesetzlichen Vorschrift in wissenschaftlicher Tiefe, gleichwohl aber in kompakter Form prägnant zu erörtern. Die Kommentierung soll breite lehrbuchartige Darstellungen vermeiden. Die wissenschaftlichen Aussagen haben in eindeutige Stellungnahmen zu münden. Die Erläuterung hat einer teleologischen Auslegung $\mathrm{zu}$ folgen, die die Wertungsgesichtspunkte herausarbeitet, aus denen sich der Gesetzessinn erschließt. In methodischer Reihenfolge geht die Auslegung vom Wortlaut aus und erläutert unter Beachtung der Entstehungsgeschichte und des Systemzusammenhangs den Gesetzeszweck. Die Auslegung hat auf diese Weise alle vier Elemente des Rechtsgewinnungsprozesses zu durchlaufen, die seit Savigny dem Kanon ordnungsgemäßer Gesetzesinterpretation entsprechen. ${ }^{12}$ Auf diesem Wege wurde bereits mit der ersten Auflage ein einheitlicher Standard der Kommentierung erreicht und seitdem konsequent beibehalten.

schichte und den Zielen des Antidiskriminierungsrechts kritisch Säcker, ZRP 2002, 286ff. sowie nunmehr eingehend Thüsing, Einl. zu Bd. I/2 der 5. Aufl. des Münchener Kommentars.

11 So ausdrücklich der SPD-Bundestagsabgeordnete O. Scholz in der Ersten Lesung des Gesetzes als Berichterstatter seiner Fraktion im Bundestag; vgl. Protokoll der 152. Sitzung des Deutschen Bundestages vom 21. 1. 2005: „Wir wollen uns einmischen - im Sinne des Anstands, den wir hier in diesem Land zu vertreten haben.".

12 Savigny, System des heutigen Römischen Rechts, Bd. I, 1840, S. 212 ff. 
Durch den in den Redaktionsrichtlinien festgeschriebenen Vorrang der teleologischen Auslegung wurde erreicht, daß alle Autoren auf ein rein formalbegriffsjuristisches Argumentieren verzichten und nicht den Anschein erwecken, man könne, wenn man einen Text nur gründlich genug lese, aus ihm für jeden behandelten Fall eine eindeutige Lösung herauslesen. Texte können semantisch nicht mehr hergeben, als in sie hineingedacht ist. Der Interpret ist keine Sphinx. Die Montesquieusche Vorstellung vom Richter als dem Mund des Gesetzes hat sich als Selbsttäuschung erwiesen. In Wahrheit geht es darum, notwendige Fortbildungen des Gesetzes, wenn der alte Normtext auf neue Problemfälle nicht mehr paßt, offen zu erörtern und sach- und systemgerechte Lösungen neu zu entwickeln; denn wir brauchen, wie Popper zu Recht festgestellt hat, beides: die Rechtssicherheit der alten Texte und die Gerechtigkeit, der sich die richterliche Rechtsfortbildung verpflichtet weiß. Es muß invariante Gesetze, die Rechtssicherheit garantieren, geben und gleichwohl, wenn nötig, Neuerungen, da das System bestehender Gesetze nie hinlänglich komplett ist. ${ }^{13}$

Linguistisch gibt es keine hermeneutisch-dialektisch zu entdeckende Wahrheit, die als Antwort auf neue Problemlagen einem Text verstehend entnommen und anderen als allein richtig mitgeteilt werden kann. Der hermeneutische Wahrheitsbegriff ist untauglich, außerhalb des Bereichs der Allgemeingültigkeit und Objektivität naturwissenschaftlicher Erkenntnis für andere verbindliche Wahrheiten festzulegen. Die Situationsbezogenheit der hermeneutischen Wahrheit ist ,so grundlegend, daß das Erkennen eines endlichen Wesens nie nach allen Seiten hin gesichert werden kann. Das Moment der Produktivität des Verstehens tritt somit in sein volles Recht und gewinnt Einlaß in die Wahrheitsproblematik. Weil das Verstehen geschichtsbedingt ist, strebt der Mensch nach neuen Einsichten, die seine Welt aufschließen und neu beleuchten können. "14 In diesem offenen Problembewußtsein haben die Autoren des Münchener Kommentars bei jeder neuen Auflage ihren Text gründlich durchdacht und so ihre Erläuterungen lebendig gehalten. Der Kommentar anerkennt die mitgestaltende Rolle des Interpreten, der ein Problem entscheiden muß, das der Gesetzgeber infolge planmäßiger oder planwidriger Unvollständigkeit des Gesetzes nicht geregelt hat.

Die schwierige Frage: „Wann soll der Richter handeln und wann soll der Kommentator dem Richter eine Rechtsfortbildung nahe legen, wenn der Gesetzgeber untätig bleibt?“", läßt sich allerdings nicht anhand einer generellen Regel entscheiden, sondern verweist jeden Autor auf seine eigene fachliche Überzeugung. Als die Lex Soraya im Bundestag gescheitert war, schuf der $B G H^{15}$ einen zivilrechtlichen Ehrenschutz entgegen § 253 BGB a.F. durch Zuerkennung von Schmerzensgeld bei erheblicher Persönlichkeitsrechtsbeeinträchtigung. Erst am 19. Juli $2002^{16}$, nach über 50 Jahren, ist das BGB durch Neufassung des $§ 253$

\footnotetext{
13 Vgl. Popper/Eccles, The Self and Its Brain, 1977, S. 25.

14 Gadamer, Hermeneutik, Bd. I, 1990, S. 270 ff.; Bd. II, S. $330 \mathrm{ff}$.

15 BGHZ 35, $363 \mathrm{ff} . ; 39,124,134 \mathrm{ff}$.

16 BGBl. I S. 2674; dazu Wagner, NJW 2002, 2049, 2053 ff.; Oetker, in: MünchKomm, § 252
} Rn. $2 \mathrm{ff}$. 
BGB an das Richterrecht angepaßt worden. Das Bundesverfassungsgericht $t^{17}$ hat die richterliche Derogation des $§ 253$ BGB gebilligt und die Verantwortlichkeit der Fachsenate für die Aktualisierung alter Texte anerkannt. ${ }^{18}$ Es bleibt damit letztlich dem „Fingerspitzengefühl“ des einzelnen Kommentators überlassen, wann er die Anpassung des alten Textes an veränderte Gegebenheiten vorschlägt und wann er auf den gesetzesabändernden Gesetzgeber verweist.

Nach dem Prinzip der inneren Kohärenz und Teleologie des Gesetzes insgesamt ist die von einem Autor vorgenommene Interpretation aus der Sicht des Gesamtwerks allerdings nur dann geglückt, ${ }^{19}$ wenn sie von einer anderen Stelle des Gesetzes nicht in Frage gestellt wird, sondern in einer vom Text her kohärenten Weise Sinn macht. ${ }^{20}$ Der Kommentar muß bemüht sein, innere Zusammenhänge zwischen den einzelnen zu erläuternden Vorschriften herzustellen, die Singularität der einzelnen Bestimmungen durch Herausarbeitung rechtsdogmatischer Funktionsgrundsätze zu bändigen und neue Normen immer wieder in das bestehende System, ggf. unter seiner immanenten Fortbildung, zu integrieren, um es durch diese Modernisierung lebendig und anpassungsfähig zu halten. Niemand hat so realistisch und eindringlich diesen Prozeß der Fortbildung des geschriebenen Rechts unter Kontrolle einer prinzipiengeleiteten Rechtsdogmatik beschrieben wie Josef Esser in seiner Monographie ,Grundsatz und Norm in der richterlichen Fortbildung des Privatrechts“. ${ }^{21}$ Der Münchener Kommentar begreift die einzelne Gesetzesvorschrift nicht als ein in sich abgeschlossenes ,,autonomes Territorium“, sondern als Teil des normübergreifenden Ganzen. Die an Telos und System des BGB gebundene Auslegung soll die Kohärenz der zivilrechtlichen Grundsätze sichern und auf diesem Wege sichtbar machen, ,wie Recht sein soll, wenn es wahres Recht sein will, streng und doch milde, gebunden und doch frei, fest und doch beweglich“. 22

Der Kommentar muß deshalb - vor allem mit Hilfe der Bandredakteure - der Gefahr begegnen, daß ein Autor nahezu jede beliebige Interpretation einer einzelnen Norm für zulässig hält und das Sinnganze des Gesetzes dabei nicht zur Kenntnis nimmt. Trotz aller interpretativen Unsicherheit, die mit der Lektüre des Gesetzestextes verbunden ist, darf ein Kommentator sich nicht zum souveränen Textproduzenten aufschwingen; er ist und bleibt auch gegenüber einer altehrwürdigen Norm Textrezipient. ${ }^{23}$ Die Interpretationskompetenz berechtigt nicht zu beliebigen Aussagen über den Gesetzesinhalt; sie verbietet das bewußte Misreading. Auslegung ist

17 BVerfGE 34, $269 \mathrm{ff}$; ferner BVerfGE 59, $104 \mathrm{ff} . ;$ 88, $103 \mathrm{ff}$; 95, $48 \mathrm{ff}$; vgl. dazu Kriele, in: Fuhrmann/Jauß/Pannenberg, Text und Applikation, 1981, S. $163 \mathrm{ff}$.

18 Allerdings nur in den Grenzen der Beachtung der wertsetzenden Bedeutung und der Schutzfunktion der Grundrechtsnormen für das Privatrecht; vgl. dazu exemplarisch BVerfGE 102, S. 347 ff.; dazu näher Säcker, WRP 2004, S. $1201 \mathrm{ff.}$

19 Vgl. Larenz, Kennzeichen geglückter Rechtsfortbildung, 1965.

20 Vgl. dazu Eco, Lektor in fabula, 3. Aufl. 1998, S. 38 ff.

21 2. Aufl. 1964; rechtsvergleichend fortführend Fikentscher, Methoden des Rechts Bd. IV, 1977.

${ }^{22}$ Windscheid, Gesammelte Reden und Abhandlungen, hrsg. von P. Oertmann, 1904, S. 48.

${ }^{23}$ Vgl. Derrida, Le Facteur de la Vérité, in: Poétique 1975, Bd. 21, S. 96 ff.; gegen Peirce, Theorie der unendlichen Interpretation. 
eine an Regeln gebundene, entziffernde ,regulierte Transformation dessen, was bereits geschrieben worden ist" ${ }^{24}$. Umberto $E c 0^{25}$ stellt daher zu Recht fest, daß in vielen Fällen niemand daran zweifelt, daß eine bestimmte Interpretation unhaltbar ist. ${ }^{26}$ Ein Jurist handelt nicht gerecht und verantwortungsbewußt, wenn er sich auf kein Recht bezieht und keine Regel für vorgegeben hält. ${ }^{27}$

Um solche Regeln als Grenzen juristischer Auslegung für den Rechtsanwendungsstab deutlich zu machen, ist es nach wie vor sinnvoll, einen methodisch vorgehenden wissenschaftlichen Kommentar zu publizieren und nicht nur einen Handkommentar, der informatorisch über die neueste Judikatur unterrichtet. Dies unterscheidet einen Großkommentar in Deutschland signifikant von Handbüchern und Kommentaren anglo-amerikanischer Prägung, die sich auf die Informationsfunktion beschränken. ${ }^{28} \mathrm{Um}$ unvertretbare Auslegungen des BGB zu vermeiden, stellt sich der Kommentar der Aufgabe, nicht nur exakt die einschlägige, aktuelle Rechtsprechung $\mathrm{zu}$ erfassen und $\mathrm{zu}$ systematisieren, sondern auch kritisch Stellung zu nehmen, wenn Gerichtsentscheidungen nach Auffassung des Autors die Grenzen zulässiger Auslegung und richterlicher Fortbildung des BGB überschreiten. Der Münchener Kommentar hat sich deshalb von Anfang an bemüht, auf der Grundlage einer zeitgemäßen, die Erkenntnisse der Linguistik und Semiotik berücksichtigenden Auslegungsmethode einen Beitrag zur Rechtsklarheit durch teleologische Bindung des Interpreten an den Sinn der kodifizierten Regeln zu leisten, ohne dabei dem Wunschtraum umfassender logisch-grammatisch vermittelter Gesetzesbindung zu unterliegen.

\section{Europäisierung des Privatrechts}

Ein neues Problem stellte sich namentlich in der vierten Auflage bei der systematischen Zusammenfügung europäischen und deutschen Privatrechts. Die zunehmende Durchdringung nationalen Rechts mit Normen EG-rechtlichen Ursprungs kompliziert den vorstehend skizzierten Prozeß der Auslegung einer Rechtsvor-

${ }^{24}$ Foucault, Archäologie des Wissens, 1981, S. 200, Haselstein, Entziffernde Hermeneutik, 1991.

${ }^{25}$ Eco, Die Grenzen der Interpretation, 1995, S. 78.

${ }^{26}$,Würde Jack the Ripper uns sagen, er habe seine Taten aufgrund einer Inspiration begangen, die ihn beim Lesen des Evangeliums überkam, so würden wir zu der Ansicht neigen, er habe das Neue Testament auf eine Weise interpretiert, die zumindest ungewöhnlich ist." Eco fügt hinzu: „Man wird angesichts der Ergebnisse seines Misreadings nicht sagen können, Jack sei das Vorbild, anhand dessen man Studenten erklären sollte, wie man mit einem Text umgeht."

27 Derrida, Gesetzeskraft, Der „mystische Grund der Autorität“, 1991, S. 48: „Umgekehrt kann er auch dann nicht als gerecht, frei und verantwortungsbewusst gelten, wenn er sich auf kein Recht, keine Regel bezieht, oder wenn er keine Regel für vorgegeben hält, die über seine Deutung hinausgeht, und deshalb die Entscheidung suspendiert, beim Unentscheidbaren stehen bleibt oder bar aller Regeln und Prinzipien improvisiert."

${ }^{28}$ Der Aktualität fühlt sich der Münchener Kommentar dadurch verpflichtet, dass er durch einen mit jeder Auflage verbundenen Loseblattband das Gesamtwerk auf dem neuesten Stand der Gesetzgebung hält und darin auch grundlegende Gerichtsentscheidungen, die von der bisherigen Judikatur abweichen, darstellt. Seit der 5. Auflage wird er ferner über www.beckonline.de ständig aktualisiert. 
schrift. Sind nationale Normen nach den vorstehend dargestellten vier Auslegungsstufen (grammatikalische, historische, systematische und teleologische Interpretation) zu interpretieren, so kann dies für Normen EG-rechtlichen Ursprungs, auch wenn diese Bestandteil der nationalen Rechtsordnung geworden sind, nicht in gleicher Weise gelten. Hier gilt der Grundsatz der eigenständigen Auslegung europäischen Rechts und der EG-rechtskonformen Auslegung nationalen Rechts. Die europäische Norm ist nicht im Wege historisch-systematischer Auslegung in die jeweilige mitgliedstaatliche Rechtsordnung zu integrieren, d.h. aus dem Kontext der nationalen Normierungen heraus auszulegen, der die europäische Vorschrift umgibt; es ist vielmehr ihre europarechtliche Grundlage zu beachten, und sie muß von dieser Grundlage her in allen Mitgliedstaaten der EG als ursprungsgleiches Recht erhalten bleiben.

Artikel 36 EGBGB gebietet ausdrücklich, bei der Auslegung der für vertragliche Schuldverhältnisse geltenden Vorschriften der Art. 27 ff. EGBGB zu berücksichtigen, daß diese entsprechend dem ihnen zugrundeliegenden völkerrechtlichen Übereinkommen einheitlich ausgelegt und angewandt werden. ${ }^{29}$ Der durch die supra- bzw. internationale Regelung erreichte Rechtsvereinheitlichungsstandard darf im Wege mitgliedstaatlicher Auslegung nicht unterschritten werden. Art. 36 EGBGB drückt damit einen an sich selbstverständlichen, allgemeinen Auslegungsgrundsatz aus, der bei den Normen supra- bzw. internationalen Ursprungs beachtet werden muß. Der hermeneutische Zirkel des Verstehens dieser vereinheitlichten Vorschriften ist nicht mehr national geprägt, sondern er ist ,europadimensional" aus der gemeinsamen Seh- und Wertungsweise der Mitgliedstaaten heraus zu definieren. Die EG-geprägte nationale Rechtsordnung ist nicht mehr allein durch die immanente Teleologie der deutschen Rechtsordnung bestimmt, sondern ist eingespannt in das Kräfteparallelprogramm mitgliedstaatlicher und EGrechtlicher Zuständigkeiten, und zwar nicht im Sinne des klassischen dualistischen Parallelismus von Völkerrecht und nationalem Recht, sondern im Sinne einer integrativen, kohärenten Gesamtordnung. ${ }^{30}$

Sind zum Beispiel als Folge der Umsetzung der Fernabsatz-Richtlinie oder der Richtlinie über mißbräuchliche Klauseln in Verbraucherverträgen Normen der $\S \S 307 \mathrm{ff}$. BGB teils nach EG-rechtlicher Teleologie und teils nach nationaler Gesetzesteleologie auszulegen, ist der Kommentar bei Zweifelsfragen um eine integrationsfreundliche, Normen nationalen und europäischen Ursprungs harmonisierende Lösung bemüht. Zwar weisen die nationalen Privatrechtsordnungen nach Zerfall des ius commune sowohl in ihrer dogmatischen Struktur als auch in ihren Resultaten zum Teil erhebliche Unterschiede auf, die eine umfassende Kodifikation des europäischen Privatrechts zum gegenwärtigen Zeitpunkt noch als verfrüht erscheinen lassen. Es entwickeln sich aber im Vorfeld einer solchen Kodifikation bereits allgemeine Prinzipien des europäischen Vertrags-, Schuld- und Kreditsicherungsrechts, die den Ausbau nationaler Sonderlösungen, die europäischen Prinzi-

\footnotetext{
${ }^{29}$ Vgl. Junker, RabelsZ 55 (1991), 674; Reinhart, RIW 1994, 445.

${ }^{30}$ Näher dazu die Einleitung zur 5. Aufl. des Münchener Kommentars zum BGB, Bd. I 2006, Rn. 137 f.
} 
pien widersprechen, zu vermeiden trachten. So hat die „Commission on European Contract Law“ (Lando-Kommission) bereits Teil I der „Principles of European Contract Law" vorgelegt. Das europäische Parlament hat die Arbeit der Lando-Kommission in seiner Entschließung zur Privatrechtsangleichung vom 6. Mai 1994 ausdrücklich begrüßt und als wichtigen Beitrag auf dem Wege zu der Ausarbeitung eines vom EG-Parlament ausdrücklich geforderten einheitlichen europäischen Gesetzbuches für das Privatrecht bezeichnet. Der Kommentar will dazu beitragen, im Rahmen des Möglichen ,durch rationale Diskussionen die Divergenzen der nationalen Rechte abzuschleifen“"31, und zwar auf der Grundlage gemeinsamer Begriffe, Institutionen, Denkformen, systematischer und ideengeschichtlicher Grundlagen.

Die Vorschriften des Bürgerlichen Rechts, die in den ersten einhundert Jahren dank aktualisierender Auslegung ihre Leistungsfähigkeit bewahren konnten, sind deshalb so fortzuentwickeln, daß die Divergenzen zu den übrigen europäischen Rechtsordnungen nicht vertieft, sondern möglichst eingeebnet werden. Für das deutsche Recht folgt daraus z.B., daß der Grundsatz von Treu und Glauben nicht zu immer stärkerer Einschränkung des Grundsatzes der Vertragstreue (etwa mittels der Lehre vom Wegfall der Geschäftsgrundlage gemäß $§ 313$ BGB) führen darf, während die Mehrzahl der europäischen Länder, insbesondere das englische Recht, der allzu weiten Auflockerung abgeschlossener Verträge durch Berufung auf Treu und Glauben kritisch gegenübersteht. Vor dem Hintergrund einer gemeinsamen europäischen Rechtskultur kommt keiner einzelnen nationalen Rechtsordnung eine Vorbild- oder Leitfunktion zu; es sind vielmehr durch eine gemeineuropäische Rechtswissenschaft die Grundlagen für eine Annäherung der Privatrechtsordnungen zu schaffen und diese dann langfristig zusammenzuführen, um in einem europäischen Binnenmarkt die wettbewerbsverzerrenden Barrieren unterschiedlicher Vertrags- und Schuldrechtsordnungen langsam abzubauen. Interpretationen, die eine nationale Rechtsordnung weiter von den sich entwickelnden Prinzipien einer europäischen Privatrechtsordnung wegführen, werden im Kommentar vermieden. P. Koschaker hat in seiner Schrift: Europa und das römische Recht, 1972, S. 1 aus rechtshistorischer Perspektive festgestellt: „Es gibt keine Sparte der Rechtswissenschaft, die so ausgesprochen europäischen Charakter trägt wie die Privatrechtswissenschaft." Wenn der Münchener Kommentar 60 Jahre nach dieser Feststellung in seiner fünften Auflage erscheint, so wird bei vielen Paragraphen deutlich werden, wie sehr sich diese Feststellung im Alltag eines Kommentators zum BGB widerspiegelt.

Der Münchener Kommentar zum BGB sieht dies, da er kein „flacher Handkommentar" sein, sondern die weitere gemeineuropäische Rechtsordnung mitgestalten will, als eine gewichtige Aufgabe an, der sich die Autoren verpflichtet fühlen. Bei der Einzelkommentierung der Vorschriften wird daher in besonderer Weise die Literatur herangezogen, die sich um die Entwicklung gemeineuropäi-

31 Zimmermann, ZOEP 1995, 732; ähnlich Tilmann, ZOEP 1993, 613 ff.; Ehricke, RabelsZ 59 (1995), 598 ff.; Grundmann, ZEuP 1996, 399 ff.; näher dazu Säcker, in: FS für R. Scholz, 2007, S. 9 ff. 
scher privatrechtlicher Prinzipien bemüht und die Lösungen anbietet, die sich in Kenntnis und in Auseinandersetzung mit den Lösungen der europäischen Nachbarrechtsordnungen ergeben, um auf diesem Wege Wertungswidersprüche zwischen nationalen Normen und Normen europarechtlichen Ursprungs möglichst zu überwinden..$^{32}$ Die richtlinienkonforme Auslegung hat daher nicht nur Bedeutung als Ergänzung der axiologischen Maxime, die rangniedrigere Rechtsquelle nach der jeweils übergeordneten ranghöheren auszulegen; ${ }^{33}$ sie hat als Auslegungsziel Vorrang vor den Auslegungszielen des nationalen Rechts. Anderenfalls würde die Einheit des Gemeinschaftsprivatrechts durch Renationalisierung im Wege der Auslegung verloren gehen.

Die redaktionellen Richtlinien haben zur Sicherung der EG-Konformität der Kommentierung seit der vierten Auflage alle Autoren auf folgende Maximen verpflichtet: Die Einbettung des deutschen Privatrechts in die europäische Rechtsordnung ist, soweit dies vom Norminhalt her geboten ist, bei der Auslegung und Fortbildung jeder Gesetzesvorschrift zu berücksichtigen. Dies erfordert bei Rechtsmaterien, zu denen Richtlinien der EG bestehen, eine richtlinienkonforme, d.h. dem Vorrang europäischer Rechtsetzung entsprechende Auslegung des deutschen Rechts; denn zum Vorrang des EG-Rechts vor dem mitgliedstaatlichen Recht gehört auch die mit dem europäischen Recht vorgegebene Auslegungsmethodik des europäischen Rechts als Einheitsrecht. Die Grundsätze der Auslegung europäischen Rechts sind Bestandteil des europäischen Rechts und haben am Vorrang europäischen Rechts Anteil. Die Kommentierung muß demgemäß unter Einbeziehung der europäischen Gerichtsbarkeit und der Literatur beachten:

a) das primäre Gemeinschaftsrecht (z.B. den Einfluß der Grundfreiheiten und der Grundrechte auf die zu erläuternde Vorschrift des BGB),

b) das sekundäre Gemeinschaftsrecht (z.B. den Bezug der zu erläuternden Norm auf Richtlinien und Rechtsverordnungen der EG),

c) das europäische Konventionsrecht (z.B. den Einfluß der Europäischen Menschenrechtskonvention auf die Auslegung der einschlägigen Normen des deutschen Rechts) einschließlich des sich entwickelnden gemeineuropäischen Rechts.

Diese Einbettung des deutschen Rechts in das europäische Recht wird unter einem eigenen Gliederungspunkt mit der Überschrift: „Gemeinschaftsrechtliche Grundlagen“ dargestellt. Durch solche Vorgaben für die Erläuterung der einzelnen Paragraphen ist es gelungen, nicht nur die innere Kohärenz des Kommentars, sondern auch seine aktuelle Weiterentwicklung über den Autorenwechsel hinaus zu sichern. Wir hoffen, daß dies auch in der fünften Auflage so bleiben wird.

\footnotetext{
32 Näher dazu am Beispiel des Sachenrechts Säcker, Vom deutschen Sachenrecht zu einem europäischen Vermögensrecht, in: FS für Georgiades, 2005, S. $359 \mathrm{ff}$.

33 Vgl. dazu bereits Burmeister, Die Verfassungsorientierung der Gesetzesauslegung, 1966; Jaenicke, ZaöR 23 (1963), $485 \mathrm{ff}$.
} 
https://doi.org/10.17104/9783406731181-405, am 26.04.2023, 13:35:48

Open Access - $(\mathrm{cc}) \mathbf{E Y}$ - http://www.beck-elibrary.de/agb 